\title{
Hemispheric asymmetries in the identification of band-pass filtered letters
}

\author{
STEPHEN D. CHRISTMAN \\ University of Toledo, Toledo, Ohio \\ FREDERICK L. KITTERLE \\ Northern Illinois University, De Kalb, Illinois \\ and \\ CHRISTOPHER L. NIEBAUER \\ University of Toledo, Toledo, Ohio
}

\begin{abstract}
Processing of band-pass filtered letters in the left versus right cerebral hemispheres (LH vs. RH) was examined. The present experiments constituted a partial replication of a study in which $\mathrm{Pe}$ terzell, Harvey, and Hardyck (1989) found no hemispheric differences in accuracy or reaction time (RT) as a function of spatial frequency. However, methodological limitations of their study (e.g., the possibility that subjects were engaged in a detection, not identification, task) may have obscured possible hemispheric differences. We addressed these problems in the present study, obtaining significant hemisphere $\times$ spatial frequency interactions for RT and $d^{\prime}$, with $\mathrm{RH}$ advantages at low frequencies and LH advantages at high frequencies; however, these effects were not large in magnitude and were often restricted to particular dependent variables, stimulus sizes, and so forth. Hemispheric differences in response bias were also found.
\end{abstract}

The hypothesis that the left versus right cerebral hemispheres in humans are specialized for the processing of high versus low spatial frequency information, respectively, has received considerable experimental attention since it was first proposed by Sergent (1982a). In their initial attempts to test this hypothesis, researchers tried to control spatial frequency content of input by varying input factors such as stimulus size, eccentricity, luminance, and exposure duration (e.g., Christman, 1987, 1990; Diehl \& McKeever, 1987; Hellige, Corwin, \& Jonsson, 1984; Sergent, 1982b). Such approaches, however, involve indirect manipulations of spatial frequency content and provide only moderate support for the spatial frequency hypothesis (Christman, 1989).

Methodologically more sophisticated tests of the hypothesis have allowed the model to be evaluated with greater precision. These tests have followed one of two approaches. First, researchers have examined processing of spectrally pure stimuli-that is, sinusoidal gratings. In earlier studies no hemispheric differences were found in the detection of high versus low spatial frequencies (e.g.,

Preparation of this paper was supported in part by a Faculty Research and Fellowship Award to S.D.C. from the University of Toledo Office of Research and by an Academic Challenge Grant to the Department of Psychology at the University of Toledo. We thank Wendy Shore, Jorge Conesa, Ray Weiner, David Peterzell, and two anonymous reviewers for helpful comments on previous versions of this manuscript. Address correspondence to S. D. Christman, Department of Psychology, University of Toledo, Toledo, OH 43606 (e-mail: schrist2@uoft02. utoledo.edu).
Blake \& Mills, 1979; Fiorentini \& Berardi, 1984; Kitterle \& Kaye, 1985; Rao, Rourke, \& Whitman, 1981). However, Kitterle, Christman, and Hellige (1990) demonstrated that hemispheric asymmetries in spatial frequency processing are found only in identification, not detection, tasks. Similarly, Christman, Kitterle, and Hellige (1991) obtained hemispheric differences in the processing of relative, as well as absolute, spatial frequency. Finally, Kitterle, Christman, and Conesa (1993) demonstrated analogous hemisphere $\times$ spatial frequency interactions in the processing of compound gratings. As discussed by Kitterle and Christman (1991), findings of visual field $X$ spatial frequency interactions in discrimination and identification, but not detection, tasks may arise from hemispheric differences in comparing the outputs of different spatial frequency channels, as opposed to hemispheric differences in sensitivity to the raw outputs of such channels.

The second approach involves digitally filtering spectrally complex stimuli to remove specific bands of spatial frequency information. Sergent (1985) employed broad-pass $(0-32 \mathrm{cpd})$ and low-pass $(0-2 \mathrm{cpd})$ faces and found an overall trend for right hemisphere $(\mathrm{RH})$ advantages for low-pass versions and left hemisphere (LH) advantages for broad-pass versions. Whitman and Keegan (1991) employed band-pass filtered faces and reported a similar visual field $X$ frequency interaction in a face matching task. Finally, Sergent (1987) employed a similar set of stimuli and obtained comparable results, although complex interactions involving task and exposure duration suggested that spatial frequency content of input 
is not the sole determinant of hemispheric asymmetries in facial processing.

Peterzell, Harvey, and Hardyck (1989) reported a series of three experiments utilizing both approaches. In Experiment 1, contrast thresholds for detection of sinusoidal gratings were examined; no hemispheric differences were found. Similarly, in Experiment 2 a gap detection task was employed as a measure of visible persistence, and again no hemispheric differences were found. These results are consistent with those of Kitterle et al. (1990), who showed that hemispheric differences are not obtained in detection tasks.

In their Experiment 3, Peterzell et al. (1989) investigated hemispheric differences in the classification of bandpass filtered letters. Letters of three different sizes were Gaussian band-pass filtered to contain 1-octave ranges of spatial frequency centered on five proximal frequencies $(1.0,2.0,4.0,8.0$, and $16.0 \mathrm{cpd})$; the size factor was included to allow examination of effects of relative frequency (e.g., a given absolute spatial frequency component of a large stimulus will have a lower relative frequency than will the same component of a smaller stimulus). They found no interaction between hemisphere and spatial frequency. The only effect was that of response bias, arising from a greater RH tendency to respond "target." Peterzell et al. concluded that previous reports of hemispheric differences in spatial frequency processing might have arisen from different hemispheric response criteria, not from true hemispheric differences in sensitivity to spatial frequency information.

The results of Experiment 3 from Peterzell et al. (1989) pose a clear question for the spatial frequency hypothesis; although Peterzell et al. employed an optimal method for manipulating spatial frequency content and employed an identification, not detection, task, they still failed to obtain interactions between spatial frequency and hemisphere. However, the design of their experiment contained potential limitations. First, their classification task involved designating the letters " $\mathrm{L}$ " and " $\mathrm{H}$ " as targets and the letters " $F$ " and " $T$ " as nontargets. Thus, nontarget letters were distinguished from targets by the presence of a horizontal crossbar running across the top edge of the stimulus; as a result, subjects might have distinguished between targets and nontargets by simply detecting whether or not a horizontal crossbar was present at the top of the stimulus. In this case, identification of the stimuli would not be necessary, and hemispheric differences would not be predicted.

Other aspects of Peterzell et al.'s (1989) design similarly limited the ability to provide an unambiguous test of the spatial frequency hypothesis. First, their analysis of the RT data collapsed across target and nontarget conditions. If this factor had significant effects on RT, collapsing across it could increase the error variance of their data. Second, although band-pass stimuli with center frequencies of 1.0 and $2.0 \mathrm{cpd}$ were employed, the results for these stimuli were not entered into the statistical analyses. This compressed the range of spatial frequencies ex- plicitly examined, which might have obscured potential hemispheric effects.

Finally, their method of stimulus presentation involved the fixation point disappearing upon stimulus onset. This raises the possibility that subjects might have occasionally engaged in express saccades and viewed stimuli with foveal vision. For example, Saslow (1967) reported that $25 \%$ of saccades had latencies of $160 \mathrm{msec}$ or less under conditions in which fixation point offset was synchronous with stimulus onset. Since Peterzell et al. (1989) employed an exposure duration of $150 \mathrm{msec}$, it is possible that subjects might have occasionally fixated the stimuli, allowing bihemispheric projection of input and thereby obscuring potential hemispheric differences.

Experiment 1 was a partial replication of Experiment 3 of Peterzell et al. (1989), in which we addressed the factors above and employed the following changes to their paradigm. First, the letters " $\mathrm{H}$ " and " $\mathrm{T}$ " were designated as targets, and " $F$ " and "L" as nontargets. Second, RT analyses included target-nontarget as a factor. Third, statistical analyses included the 1.0- and 2.0-cpd stimuli. Fourth, the fixation point remained present throughout stimulus presentation to reduce the possibility of express saccades.

Finally, the total number of trials administered to subjects was reduced in our replication, for two reasons. Peterzell et al. (1989) employed a total of 108 different stimuli, including (1) stimuli of three different sizes, (2) unfiltered letters, and (3) two sets of filtered letters, one set unmatched for contrast and the other matched for equal contrast. They found no hemispheric differences with the unfiltered letters, nor as a function of either letter size or matched versus unmatched contrast; therefore, in the present study we employed only filtered, contrastmatched stimuli of two sizes, corresponding to Peterzell et al.'s large and small letter sizes. Additionally, Peterzell et al.'s Experiment 3 involved a total of 2,160 trials per subject. This raised the possibility of fatigue and/or practice effects on the behalf of subjects, further obscuring possible hemispheric differences in spatial frequency processing.

\section{EXPERIMENT 1}

\section{Method}

Subjects. Twenty University of Toledo undergraduates served as subjects. All were right-handed and had no left-handed relatives, as assessed by a brief handedness inventory (Oldfield, 1971). All subjects had normal or corrected-to-normal vision.

Stimuli. Stimuli consisted of the letters L, H, T, and F in the Avant Garde font, a sans serif font similar to the Futura Medium font employed by Peterzell et al. (1989). The stimuli subtended $2.0^{\circ}$ or $0.5^{\circ}$ in height. Following the procedures of Peterzell et al., the stimuli were band-pass filtered to contain 1 octave ranges of spatial frequency. Filtering was done with the NIH Image program, version $1.28 \mathrm{~b}$. First, two-dimensional fast Fourier transforms were computed. Subsequent frequency spectra were then band-pass filtered; these filters were isotropic in orientation with 1 -octave Gaussian frequency profiles symmetrical in terms of $\log$ spatial frequency. Five filters with center frequencies of $1.0,2.0,4.0,8.0$, and $16.0 \mathrm{cpd}$ of visual angle were employed. The large letters were filtered with all five filters; the small letters were filtered with the 4.0-, 8.0-, and 16.0-cpd filters. 
Owing to constraints in the method of stimulus generation, the contrast values of 0.30 employed by Peterzell et al. (1989) were not matched; rather, stimuli in the present study had matched peak-topeak contrasts of 0.60 . However, evidence for hemispheric differences as a function of contrast level is equivocal at best: in a review of five studies, Christman (1989) found that increases in contrast affected both hemispheres equally in three, improved RH performance relative to $\mathrm{LH}$ in one, and improved $\mathrm{LH}$ performance relative to $\mathrm{RH}$ in the fifth. More directly, there is no evidence for differential effects of contrast level on hemispheric processing of spatial frequency information (see, e.g., Kitterle et al., 1990; Kitterle \& Kaye, 1985; Previc, 1982). Thus, the higher contrast employed in the present study should have had little or no effect on the nature of the predicted visual field effects.

A related concern involves the presence of distortions in contrast arising from the nonlinear relationship between the gray level specified in the digital filtering algorithm and the actual luminance value displayed on the monitor; in the present study, we did not apply a "gamma" correction. However, this distortion is most severe at very low luminance/gray level values, which we did not utilize in the present study. We calculated luminance as a function of specified gray level for the entire range of 256 gray levels, and the total amount of distortion amounted to an average of $3.2 \%$ for the range of luminance values employed (maximum luminance $=39.4 \mathrm{~cd} / \mathrm{m}^{2}$, minimum luminance $=9.5 \mathrm{~cd} / \mathrm{m}^{2}$ ). Given the aforementioned discussion of the lack of effects of contrast on hemispheric differences in spatial frequency processing, the amount of distortion in the stimuli used here likely had negligible effects on hemispheric processing.

Apparatus. All stimuli were generated, filtered, and presented on a Macintosh II computer with a high-resolution monitor and an Apple $8 \cdot 24 \mathrm{GC}$ graphics accelerator card. Stimulus presentation and response timing were under the control of the Reaction Time module of the MacLaboratory program, version 2.1 (Chute, 1988). Peterzell et al. (1989) did not specify luminance values for their Experiment 3; consequently, the present study is unable to replicate this factor. The stimuli in the present experiment had a space-averaged luminance of $18.4 \mathrm{~cd} / \mathrm{m}^{2}$, and were presented on a gray background matched for average luminance.

Procedure. Subjects sat at a desk in front of the computer, viewing the monitor from a distance of $70 \mathrm{~cm}$. The fixation point consisted of a small white " $x$ " and was present throughout each trial, which began with a 200 -msec warning tone, followed $600 \mathrm{msec}$ later by presentation of a stimulus to the left visual field (LVF) or right visual field (RVF). The stimuli were positioned with their innermost edge $1.5^{\circ}$ to the left or right of the fixation point. " $\mathrm{H}$ " and " $T$ " were designated as target letters and "F" and " $L$ " as nontarget letters. The subjects were instructed to press a key with the index finger of one hand if the stimulus was a target and to press another key with the other index finger if the stimulus was a nontarget. Half of the subjects responded to targets with their left hand and half with their right. Following the procedures of Peterzell et al. (1989), different stimulus sizes were run in separate blocks. There were 240 small letter trials, yielded by the factorial combination of 4 letters $\times 3$ filters $\times 2$ visual fields $\times 10$ replications and presented in two blocks of 120 trials. There were 400 large letter trials, yielded by the factorial combination of 4 letters $\times 5$ filters $\times 2$ visual fields $\times 10$ replications and presented in two blocks of 120 trials and one block of 160 trials. Order of conditions was counterbalanced across subjects. All subjects were given a block of 20 practice trials before participating in their first block of experimental trials.

\section{Results}

Response measures based on RT and accuracy were analyzed. RT measures were based on subjects' median RTs for correct responses. Raw accuracy scores, as Peterzell et al. (1989) point out, are known to be influenced by decision criteria. Consequently, signal detection procedures were employed to transform hit and false alarm rates into a sensitivity measure $\left(d^{\prime}\right)$ and a response bias measure $(\beta)$. A $d^{\prime}$ of 0 indicates a complete lack of sensitivity, with larger values indicating greater accuracy. $\beta$ ranges from 0 to $\infty$, with $\beta$ values less than one indicating a bias to respond "target," $\beta$ values greater than one indicating a bias to respond "nontarget", and a $\beta$ of 1.0 indicating a lack of response bias. Since the distribution of $\beta$ is skewed, analyses were performed on the log transform of $\beta$. The data for the three dependent variables are graphed in Figure 1

Since the small stimuli involved three levels of filtering, while the large stimuli involved five, analyses were performed in two stages. First, separate analyses were carried out for each letter size. Second, combined analyses were conducted, based on the three levels of filtering (i.e., 4.0, 8.0, and $16.0 \mathrm{cpd}$ ) that both letter sets shared in common.

RT analyses. Four-way analyses of variance (ANOVAs) were carried out for the large- and small-letter conditions, with hand (left vs. right) assigned to "target" responses as a between-subjects factor, and visual field (LVF vs. RVF), spatial frequency $(1.0,2.0,4.0,8.0$, and $16.0 \mathrm{cpd}$ for the large letters; $4.0,8.0$, and $16.0 \mathrm{cpd}$ for the small letters), and target (target vs. nontarget) as within-subjects factors. For the large-letter condition, main effects of spatial frequency $[F(4,72)=27.36, p<$ $.001]$ and target $[F(1,18)=13.76, p<.002]$ were obtained, arising from faster RTs to intermediate frequencies and to targets. A target $X$ hand of response interaction was also obtained $[F(1,18)=6.74, p<.02]$, arising from faster RTs to targets than to nontargets when subjects responded to targets with their right hand. A visual field $\times$ spatial frequency interaction $[F(4,72)=2.54$, $p<.05]$ arose from a nonsignificant LVF advantage at $1 \mathrm{cpd}[F(1,19)=2.05, p<.17]$, no visual field differences at 2 and $4 \mathrm{cpd}(F \mathrm{~s}<1)$, and RVF advantages at $8 \mathrm{cpd}[F(1,19)=5.49, p<.03]$ and $16 \mathrm{cpd}[F(1,19)=$ $5.70, p<.03]$. Finally, the interaction of visual field, spatial frequency, and target narrowly missed significance $[F(4,72)=2.40, p<.06]$, arising from the fact that the spatial frequency $\times$ visual field interaction was more pronounced for targets than for nontargets. All other $p$ values were 30 or greater. Standard errors ranged from 36.9 to 48.2 .

Similar results were obtained for the small-letter condition. There were main effects of spatial frequency $[F(2,36)=10.65, p<.001]$ and target $[F(1,18)=10.96$, $p<.004]$, reflecting the same effects found for the largeletter condition. Similarly, a hand of response $\times$ target interaction was obtained $[F(1,18)=5.00, p<.04]$, reflecting faster RTs to targets than to nontargets for subjects responding to targets with the right hand. A spatial frequency $\times$ target interaction was obtained $[F(2,36)=$ $3.98, p<.03$ ], arising from faster RTs to targets at $8.0 \mathrm{cpd}$ and to nontargets at $4.0 \mathrm{cpd}$. A marginal interaction between spatial frequency and hand of response $[F(2,36)=2.80, p<.08]$ reflected faster RTs at 8.0 and $16.0 \mathrm{cpd}$ for subjects responding with the right hand. Finally, a spatial frequency $\times$ visual field interaction $[F(2,36)=4.18, p<.03]$ arose from a lack of visual field differences at $4 \mathrm{cpd}(F<1)$, an LVF advantage at $8 \mathrm{cpd}$ $[F(1,19)=8.10, p<.01]$, and no visual field differences 


\section{IARGE}
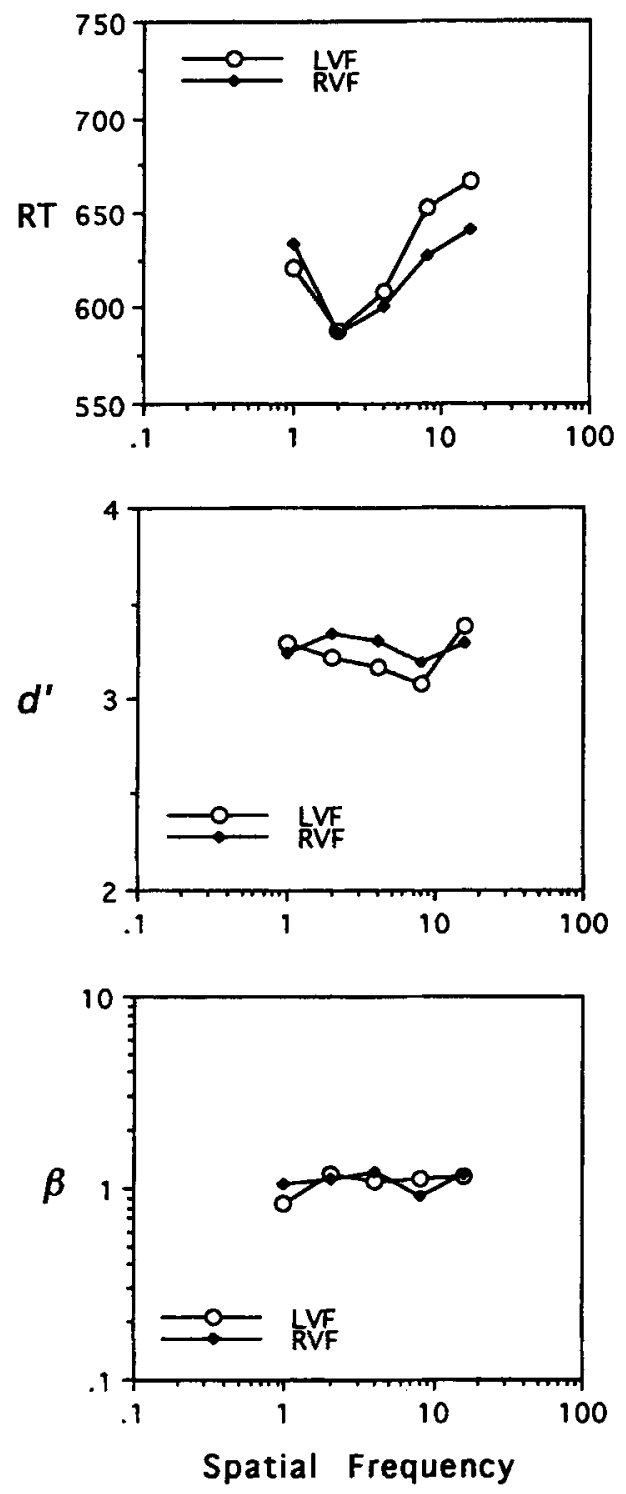

SMALL
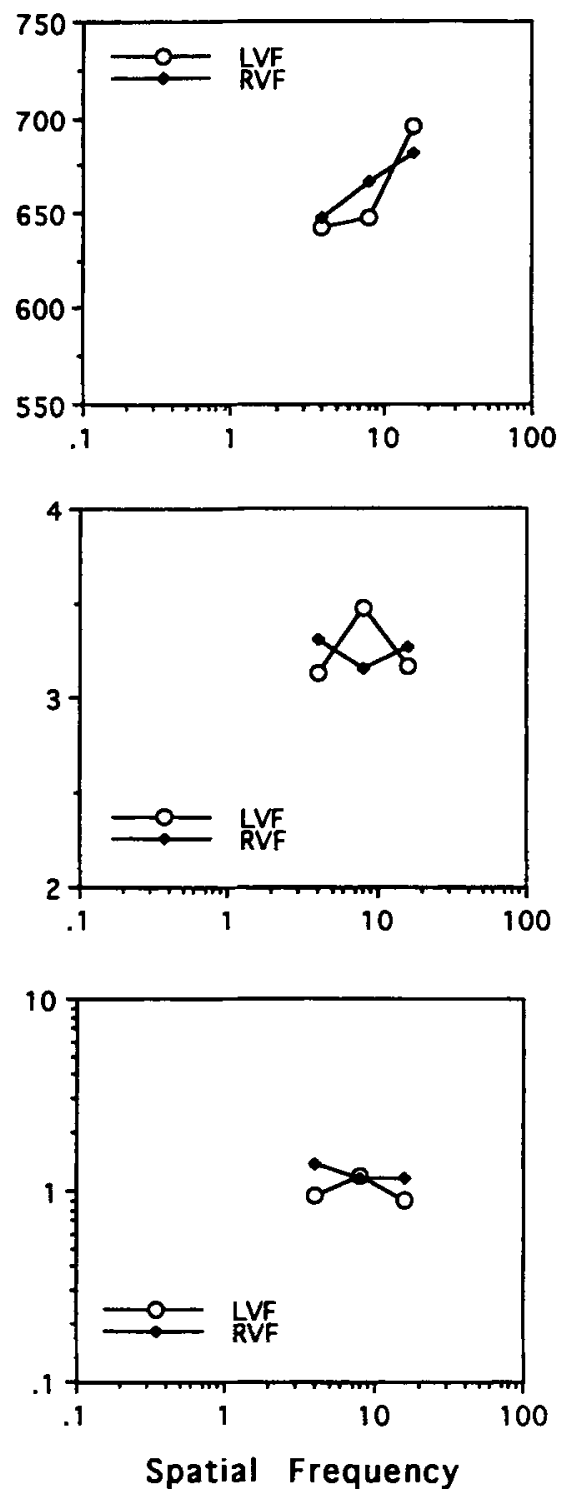

Figure 1. Performance from Experiment 1 on the letter-classification task in the LVF versus RVF as a function of filter center spatial frequency (cycles per degree) for the large-letter (left column) and small-letter (right column) sets. Top row: reaction time (RT) in milliseconds. Middle row: accuracy data $\left(d^{\prime}\right)$. Bottom row: response bias $(\beta)$. Each point is the mean of 20 subjects.

at $16 \mathrm{cpd}[F(1,19)=1.92, p<.19]$. All other effects were nonsignificant, with $p$ values of .21 or greater. Standard errors ranged from 33.6 to 42.8 .

A five-way ANOVA performed on the combined RT data (with size added as the fifth factor) allowed explicit examination of the effects of stimulus size. All significant effects not involving size simply echoed effects discussed above and will not be detailed, except to point out that the predicted spatial frequency $\times$ visual field interaction was again significant $[F(2,36)=5.61, p<.03]$; this reflected no visual field differences at 4.0 and $8.0 \mathrm{cpd}$ $(F \mathrm{~S}<1)$ and an RVF advantage at $16 \mathrm{cpd}[F(1,19)=11.26$, $p<.003]$. The only effect involving size was a size $\times$ vi- sual field interaction $[F(1,19)=5.61, p<.03]$, arising from the presence of an RVF advantage for large letters and no visual field differences for small letters.

Signal detection analyses. Three-way ANOVAs were carried out on the $d^{\prime}$ measure of accuracy for the largeand small-letter conditions separately, with hand of response as a between-subjects factor, and visual field and spatial frequency as within-subjects factors. There were no significant main effects or interactions for the largeletter set. For the small-letter set, the only effect to approach significance was a trend toward an interaction between spatial frequency $\times$ visual field $[F(2,36)=3.23$, $p=.051]$. This arose from no visual field differences at 
$4 \operatorname{cpd}[F(1,19)=1.95, p<.20]$, a nonsignificant LVF advantage at $8 \mathrm{cpd}[F(1,19)=3.07, p<.11]$, and no differences at $16 \mathrm{cpd}(F<1)$; all other $p$ values were greater than .18. Analyses of the combined data (with size added as a fourth factor) yielded two significant effects. First, a main effect of hand of response $[F(1,18)=4.85, p<$ $.05]$ arose from the presence of higher $d^{\prime}$ scores for subjects who responded to targets with the right hand. Second, there was a size $\times$ visual field $\times$ spatial frequency interaction $[F(2,36)=4.05, p<.03]$, reflecting the fact that the visual field $\times$ spatial frequency interaction was present in the small-letter set only. Standard errors ranged from 0.163 to 0.207 .

Three-way ANOVAs were also performed on $\log$ transforms of the $\beta$ measure. For the large-letter condition, the only significant effect was that of spatial frequency $[F(4,72)=2.67, p<.04]$, reflecting a trend for $\beta$ to increase with increased spatial frequency. For the small-letter condition, the only significant effect was a main effect of visual field $[F(1,18)=4.47, p<.05]$, reflecting a trend for lower $\beta$ values in the LVF (i.e., a greater bias to respond "target" for LVF trials). Standard errors ranged from 0.054 to 0.166 . Analyses of the combined data revealed two significant interactions: spatial frequency $\times$ visual field $[F(2,36)=5.00, p<.02]$, arising from the presence of relatively lower $\beta$ scores in the $\mathrm{LVF}$ at 4 and $16 \mathrm{cpd}$ and in the RVF at $8 \mathrm{cpd}$, and size $\times$ visual field $\times$ hand of response $[F(1,18)=7.97, p<$ $.02]$, arising from the fact that $\beta$ scores were generally lower on LVF trials than on RVF trials except in the case of subjects in the large-letter set who responded to targets with the right hand.

\section{EXPERIMENT 2}

Although Experiment 1 yielded visual field $\times$ spatial frequency interactions in RT that were roughly consistent with the spatial frequency hypothesis, interpretation of why we obtained such effects, and why Peterzell et al. (1989) did not, remained ambiguous because of the numerous changes in methodological factors from Peterzell et al.'s original design (viz., in target identity, number of trials, stimulus contrast, temporal course of fixation point). We were inclined to interpret our results primarily in terms of the change in target identity, necessitating identification of the targets, it was possible that some other factor(s) determined our results. Consequently, we undertook a second experiment, in which we exactly replicated our Experiment 1 except for using the target/nontarget groupings originally employed by Peterzell et al. To the extent that the visual field $\times$ spatial frequency interaction found in Experiment 1 arose from the target/nontarget reclassification, we expected not to obtain the field $\times$ frequency interaction in this second experiment.

\section{Method}

All details of this experiment were identical to those of Experiment 1 , with the exception that, following the procedures of Peterzell et al. (1989), the letters " $H$ " and "L" were designated as targets, and the letters " $F$ " and " $T$ " were designated nontargets. As in Experiment 1, 20 right-handed subjects without left-handed relatives served as observers.

\section{Results}

RT analyses. Four-way ANOVAs were carried out for the large- and small-letter conditions, with hand (left vs. right) assigned to "target" responses as a between-subjects factor, and visual field (LVF vs. RVF), spatial frequency $(1.0,2.0,4.0,8.0$, and $16.0 \mathrm{cpd}$ for the large letters; $4.0,8.0$, and $16.0 \mathrm{cpd}$ for the small letters) and target (target vs. nontarget) as within-subjects factors. The data for all three independent variables are shown in Figure 2. For the large-letter condition, a main effect of spatial frequency was obtained $[F(4,72)=7.97, p<$ $.001]$, arising from faster RTs to intermediate frequencies. A marginal interaction between spatial frequency and hand of response $[F(4,72)=2.44, p<.06]$ reflected faster RTs to targets at all spatial frequencies except at 1.0 and $2.0 \mathrm{cpd}$ for subjects responding to targets with the left hand. There was also a spatial frequency $\times$ target interaction $[F(4,18)=3.17, p<.02]$; however, this effect was qualified by a three-way interaction between spatial frequency, target, and hand of response. This interaction reflected the presence of faster RTs to targets than to nontargets at all spatial frequencies for subjects responding to targets with the right hand; for subjects responding to targets with the left hand, however, RTs were faster to nontargets at the two lowest spatial frequencies. Finally, there was a spatial frequency $\times$ visual field $\times$ target interaction $[F(4,72)=3.72, p<.01]$. For targets, there were no visual field differences at $1.0 \mathrm{cpd}$ $(F<1), 2.0 \mathrm{cpd}(F<1), 4.0 \mathrm{cpd}[F(1,18)=1.91, p>$ $.18]$, and $8 \mathrm{cpd}(F<1)$; there was a nonsignificant LVF advantage at $16 \mathrm{cpd}[F(1,18)=3.40, p<.09]$. Nontargets yielded a nonsignificant LVF advantage at $1.0 \mathrm{cpd}$ $[F(1,18)=3.94, p<.07]$, no visual field differences at $2.0 \mathrm{cpd}(F<1), 4.0 \mathrm{cpd}[F(1,18)=1.28, p>.27]$, and $8.0 \mathrm{cpd}(F<1)$, and a nonsignificant RVF advantage at $16.0 \mathrm{cpd}[F(1,18)=3.82, p<.065]$. All other effects were nonsignificant, with $p$ values of 12 or greater. Standard errors ranged from 29.4 to 46.4 .

For the small-letter condition, there was again a main effect of spatial frequency $[F(2,36)=11.60, p<.001]$, arising from longer RTs to $16.0 \mathrm{cpd}$. There was also a visual field $\times$ target interaction $[F(1,18)=5.33, p<.04]$, which was qualified by a visual field $X$ target $X$ hand of response interaction $[F(1,18)=11.30, p<.005]$. This interaction reflected the fact that subjects responding to targets with their left hand exhibited LVF advantages for targets and RVF advantages for nontargets; there were no visual field differences for subjects responding to targets with their right hand. The spatial frequency $\times$ visual field interaction was not significant $[F(2,36)=2.48, p<$ $.10]$, although the simple effects were in the direction predicted by the spatial frequency hypothesis, with a nominal 18-msec LVF advantage at $4 \mathrm{cpd}$ and a nominal $12-$ msec RVF advantage at $16 \mathrm{cpd}$. All other $p$ values were .23 or higher. Standard errors ranged from 33.4 to 41.5 . 



Figure 2. Performance from Experiment 2 on the letter-classification task in the LVF versus RVF as a function of filter center spatial frequency (cycles per degree) for the large-letter (left column) and small-letter (right column) sets. Top row: reaction time (RT) in milliseconds. Middle row: accuracy data $\left(d^{\prime}\right)$. Bottom row: response bias $(\beta)$. Each point is the mean of 20 subjects.

A four-way ANOVA performed on the combined RT data (with size added as the fourth factor) allowed explicit examination of the effects of stimulus size at 4.0, 8.0 , and $16.0 \mathrm{cpd}$. There was a main effect of spatial frequency $[F(2,36)=10.66, p<.001]$, reflecting fastest RTs at $4.0 \mathrm{cpd}$. There was also a target $\times$ visual field interaction $[F(1,18)=7.35, p<.02]$, arising from the presence of LVF versus RVF advantages for targets versus nontargets, respectively. A size $x$ target $\times$ visual field $\times$ hand of response interaction $[F(1,18)=9.56, p<.01]$ reflected the fact that the aforementioned target $X$ visual field $\times$ hand of response interaction was present for small stimuli only. Similarly, a size $\times$ spatial frequency $\times$ visual field $\times$ target interaction $[F(2,36)=6.19, p<$ $.005]$ reflected the fact that the three-way interaction between spatial frequency, visual field, and target was found for large stimuli only.

Signal detection analyses. Three-way ANOVAs were carried out on the $d^{\prime}$ measure of accuracy for the largeand small-letter sets separately, with visual field and spatial frequency as within-subjects factors and hand of response as a within-subjects factor. There were no significant effects for either the large- or small-letter sets, although the small-letter set did yield a trend toward a 
spatial frequency $\times$ visual field interaction $[F(2,36)=$ $2.44, p<.10]$, arising from trends toward higher $d^{\prime}$ values in the LVF at $4.0 \mathrm{cpd}(p<.07)$ and in the RVF at $16.0 \mathrm{cpd}(p<.18)$. All other $p$ values were greater than .20. Standard errors ranged from 0.127 to 0.217 . Analyses of the combined data (with size added as a fourth factor) yielded a single effect: a visual field $\times$ spatial frequency interaction $[F(2,36)=3.88, p<.03]$, arising from the presence of a marginal LVF advantage at $4 \mathrm{cpd}$ $[F(1,19)=4.23, p=.054]$, no differences at $8 \mathrm{cpd}$ $(F<1)$, and a marginal RVF advantage at $16 \mathrm{cpd}[F(1,19)$ $=3.84, p=.065]$.

Three-way ANOVAs were performed on log transforms of the $\beta$ measure. There were no main effects or interactions for the small letter set (all $F \mathrm{~s}<1$ ). For the large-letter set, there was a significant effect of hand of response $[F(1,18)=4.66, p<.05]$, reflecting the presence of a bias to respond "nontarget" for responding to targets with the right hand and a bias to respond "target" for responding to targets with the left hand. There was also a nonsignificant effect of visual field $[F(1,18)=$ $3.05, p<.10]$, reflecting biases to respond "target" versus "nontarget" on LVF versus RVF trials, respectively. Standard errors ranged from 0.029 to 0.098 . Analyses of the combined data yielded no significant effects.

\section{DISCUSSION}

The present experiments provide a complicated extension to the results reported by Peterzell et al. (1989). On the one hand, both of our experiments yielded interactions involving spatial frequency and visual field: in the RT data from Experiment 1, the large-letter set yielded marginal LVF advantages at lower frequencies and significant RVF advantages at higher frequencies, whereas the results of Experiment 2 yielded similar trends in RT for the small-letter set and for nontargets in the large-letter set, as well as in the combined $d^{\prime}$ data collapsed across size. On the other hand, the simple effects underlying these interactions were often not significant, suggesting that these effects are, at best, of rather small magnitude, and, at worst, may not be very reliable; indeed, if the probability of making a Type I error had been set at .01, as was done in the study by Peterzell et al., the only significant visual field effect in our data would have been the visual field $\times$ spatial frequency $\times$ target interaction in RT for the large-letter set in Experiment 2. Furthermore, the interactions between spatial frequency and visual field in the $d^{\prime}$ and RT data in Experiment 1 for small letters, with no visual field differences at 4 and $16 \mathrm{cpd}$ and an LVF advantage at $8 \mathrm{cpd}$, are not easily accounted for by the spatial frequency hypothesis. Finally, the fact that the visual field effects were generally restricted to certain sizes of letters and/or nontargets only also indicates that the influence of spatial frequency content on visual field differences is readily modulated by other input characteristics. In this sense, our results, in conjunction with those reported by Peterzell et al., suggest that the spatial frequency hypothesis, as originally formulated by Sergent (1982a), can be rejected, because it predicts straight forward hemisphere $x$ frequency interactions. Nonetheless, the present results do indicate that spatial frequency content influences hemispheric processing, although these effects are not as robust and consistent for band-pass filtered stimuli as opposed to sinusoidal stimuli. Future research should attempt to more precisely delineate the conditions under which hemisphere $x$ frequency interactions are or are not obtained.

Two important issues are raised by the present results. The first is the question of why we obtained visual field $X$ spatial frequency interactions (granting that they were weak and inconsistent), whereas Peterzell et al. (1989) did not. Given that we obtained visual field $\times$ frequency effects in both experiments suggests that the null results reported by Peterzell et al. did not reflect the fact that their target/nontarget classification may have allowed subjects to perform the task on the basis of a feature detection strategy (indeed, one might argue that letter classification in our first experiment could be performed on the basis of symmetry detection, since our targets, " $\mathrm{H}$ " and " $T$," were symmetrical, whereas nontargets were not). A more likely possibility involves the different number of trials employed in the two studies. Our study involved 660 trials per subject; Peterzell et al.'s study involved 2,160 trials per subject. The extensive practice that subjects gained over the course of Peterzell et al.'s study may have allowed both hemispheres to become proficient at the task, thereby attenuating hemispheric differences. For example, perhaps subjects were able, with practice, to learn how to perform the letter classification task on the basis of feature detection, but this strategy takes more than 660 trials to develop.

A second issue concerns the contrast between the weak nature of the visual field $x$ spatial frequency interactions obtained in the present study with digitally filtered letters and the strong visual field $x$ spatial frequency interactions obtained with sinusoidal gratings in earlier studies from our lab (e.g., Christman et al., 1991; Kitterle et al., 1990). An important factor here concerns the fact that hemispheric asymmetries are typically multiply determined by numerous factors (cf. Hellige, 1993). In previous studies done with sinusoidal gratings, the simple nature of the stimuli forced subjects to perform the task on the basis of spatial frequency content alone. In the present letter classification task, however, numerous other input factors could be used in the process of target classification (e.g., phase, orientation, local luminance cues, etc.); these other factors may have attenuated underlying hemispheric differences in processing spatial frequency per se. This speculation is reinforced by the conclusion reached by Christman (1989) in a literature review of 79 laterality experiments in which input characteristics related to spatial frequency content were manipulated. His review indicated that manipulations of spatial frequency content of complex stimuli (e.g., faces, letters, words, etc.) are only moderately successful at inducing visual field $x$ spatial frequency interactions in the direction predicted by the spatial frequency hypothesis; that is, $57 \%$ of the studies reviewed yielded interactions in the predicted direction, while $32 \%$ yielded no interaction and $11 \%$ yielded interactions in the opposite direction. In summary, these findings indicate that the role of spatial frequency content in the identification of complex input is only one of many factors that determine patterns of hemispheric asymmetry.

With regard to response bias, our results are consistent with those reported by Peterzell et al. (1989), who found that the LVF versus RVF exhibited biases to respond "target" versus "nontarget," respectively. We found similar trends in both studies, although this effect was significant only for the small letters in Experiment 1 . Moreover, an interaction between visual field and spatial frequency was obtained for the $\beta$ measure in the small-letter set from Experiment 1. Peterzell (1991) argued that, since most laterality studies have not used independent measures of accuracy and response bias, previous reports of visual field $\times$ spatial frequency interactions may have arisen from hemispheric differences in response bias, rather than hemispheric differences in accuracy or sensitivity. The present results (at least for the small letters) are partly compatible with such a hypothesis: the presence of lower $\beta$ values in the LVF versus RVF for 4- versus 8-cpd stimuli could possibly lead to analogous RT and/or accuracy advantages; for example, if there was a bias to respond "target" in the LVF versus RVF at low versus high frequencies, respectively, this might have led to "fast guesses" with short RTs and responses scored as correct (even if the subject had not actually identified the stimulus).

However, a number of factors suggest that it is unlikely that response biases could solely explain previous reports of visual field $x$ spatial frequency interactions. First, visual field $X$ spatial frequency interactions for RT data obtained in the present study held for both letter sets, even though the visual field $\times$ spatial frequency interaction for the $\beta$ measure was obtained for the small-letter set only. Second, as Christman (1993) pointed out, most previous reports of hemispheric differences in spatial frequency processing have been based on RT, a dependent measure less susceptible to response biases. Nonetheless, in conjunction with other reports of hemispheric differences in re- 
sponse bias (e.g., Chiarello, Senehi, \& Soulier, 1986; Kitterle et al., 1993; Peterzell et al., 1989), the present study demonstrates the importance of taking potential response biases into account when interpreting hemispheric differences in performance

Two discrepancies between the present results and those reported by Peterzell et al. (1989) bear mention. First, $d^{\prime}$ values in Peterzell et al.'s study ranged from about 1.0 to 2.8 , whereas in the present study they ranged from 3.0 to 3.5. This may have arisen from two differences between the studies: (1) the present total of 660 trials as opposed to Peterzell et al.'s 2,160 trials, which raises the possibility of fatigue on behalf of Peterzell et al.'s subjects; (2) the present contrast levels, which were higher than those in Peterzell et al.'s study, and which would have benefited performance. A second discrepancy concerns the specific $d$ values obtained for the 16-cpd stimuli: the $d^{\prime}$ values for these stimuli in Peterzell et al.'s study were around 1.0 , whereas in our study they were around 3.3. This probably arose from the higher contrast values employed here. Under viewing conditions comparable to those used by Peterzell et al. (1989), Kitterle et al. (1990) demonstrated that the contrast threshold for 12-cpd stimuli was about 0.30 ; the contrast threshold was well below 0.10 for frequencies between 1 and $10 \mathrm{cpd}$. This indicates that the 16-cpd stimuli in Peterzell et al.'s study were either at or barely above threshold. The present contrast level of 0.60 , therefore may have preferentially benefited processing of the 16-cpd stimulus.

In conclusion, the present study provides further confirmation of hemispheric differences in the processing of spatial frequency, but with the caveat that such differences appear to manifest themselves less robustly in the processing of complex patterns as opposed to sinusoida stimuli. Furthermore, these hemispheric differences in response bias (as well as visual field $X$ spatial frequency interactions in response bias) highlight the need for the use of signal detection procedures to dissociate sensitivity and bias effects in future tests of the spatial frequency hypothesis (and other hemispheric differences in general).

\section{REFERENCES}

BLAKE, R., \& MILLS, J. (1979). Pattern and flicker detection examined in terms of nasal-temporal division of the retina. Perception, 8, 549-555

Chiarello, C., Senehi, J., \& Soulier, M. (1986). Viewing conditions and hemisphere asymmetry for the lexical decision. Neuropsychologia, 24, 521-529.

Christman, S. D. (1987). Effects of perceptual quality on hemispheric asymmetries in visible persistence. Perception \& Psychophysics $41,367-374$

Christman, S. [D.] (1989). Perceptual characteristics in visual laterality research. Brain \& Cognition, 11, 238-257.

Christman, S. [D.] (1990). Effects of luminance and blur on hemispheric asymmetries in temporal integration. Neuropsychologia, 28, 361-374.

Christman, S. [D.] (1993). On the complex relation between perceptual characteristics and hemispheric asymmetry. Brain \& Cognition, 21, 123-129

Christman, S. [D.], Kitterle, F. L., \& Hellige, J. [B.] (1991), Hemispheric asymmetry in the processing of absolute versus relative spatial frequency. Brain \& Cognition, 16, 62-73.

Chute, D. (1988). Reaction time. In MacLaboratory for psychology: Part 2. Santa Barbara, CA: Kinko's Academic Courseware Exchange.

DieHL, J. A., \& McKeEver, W. F. (1987). Absence of exposure time influence on lateralized face recognition and object naming latency tasks. Brain \& Cognition, 6, 347-359.
Fiorentini, A., \& Berardi, N. (1984). Right-hemisphere superiority in the discrimination of spatial phase. Perception, 13, 695-708.

Hellige, J. B. (1993). Hemispheric asymmetry: What's right and what's left. Cambridge, MA: Harvard University Press.

Hellige, J. B., Corwin, W. H., Jonsson, J. E. (1984). Effects of perceptual quality on the processing of human faces presented to the left and right cerebral hemispheres. Journal of Experimental Psychologv: Human Perception \& Performance, 10, 90-107.

KitTerle, F. L., \& Christman, S. [D.] (1991). Hemispheric symmetries and asymmetries in the processing of sine-wave gratings. In F. L. Kitterle (Ed.), Advances in cerebral laterality research: Data and theory (pp. 201-224). Hillsdale, NJ: Erlbaum.

Kitterle, F. L., Christman, S. [D.], \& Conesa, J. (1993). Hemispheric differences in the interference among components of compound gratings. Perception \& Psychophysics, 54, 785-793.

Kitterle, F. L., Christman, S. [D.], \& Hellige, J. B. (1990). Hemispheric differences are found in the identification, but not the detection, of low versus high spatial frequencies. Perception \& Psychophysics, 48, 297-306.

KitTERLE, F. L., \& KAYE, R. S. (1985). Hemispheric symmetry in contrast and orientation sensitivity. Perception \& Psychophysics, 37, $391-396$.

OLDFIELD, R. (1971). The assessment and analysis of handedness: The Edinburgh Inventory. Neuropsychologia, 9, 97-113.

Peterzell, D. H. (1991). On the nonrelation between spatial frequency and cerebral hemispheric competence. Brain \& Cognition, $15,62-68$.

Peterzell, D. H., Harvey, L. O., JR., \& Hardyck, C. D. (1989). Spatial frequencies and the cerebral hemispheres: Contrast sensitivity, visible persistence, and letter classification. Perception \& Psychophysics, 46, 443-455.

Previc, F. H. (1982). Visual pattern recognition in the cerebral hemispheres: The role of spatial filtering. Perceptual \& Motor Skills, 55, 1319-1326.

RaO, S. M., Rourke, D., \& Whitman, R. D. (1981). Spatio-temporal discrimination of frequency in the right and left visual fields: A preliminary report. Perceptual \& Motor Skills, 53, 311-316.

SASLow, M. G. (1967). Latency for saccadic eye movement. Journal of the Optical Society of America, 57, 1030-1033.

SERGENT, J. (1982a). The cerebral balance of power: Confrontation or cooperation? Journal of Experimental Psychology: Human Perception \& Performance, 8, 253-272.

SERGENT, J. (1982b). Theoretical and methodological consequences of variations in exposure duration in laterality studies. Perception \& Psychophysics, 31, 451-461.

SeRGENT, J. (1985). Influence of task and input factors on hemispheric involvement in face processing. Journal of Experimental Psychology: Human Perception \& Performance, 11, 846-861.

SERGENT, J. (1987). Failures to confirm the spatial-frequency hypothesis: Fatal blow or healthy complication? Canadian Journal of Psychology, 41, 412-428.

WhitMan, R. D., \& KeEGAN, J. F. (1991). Lateralization of facial processing: A spatial frequency model. International Journal of Neuroscience, 60, 177-185.

(Manuscript received June 30, 1994; revision accepted for publication April 12, 1996.) 